\title{
Eradicate Coronavirus by blocking replication, counteracting its defense system
}

\author{
Dr. Kira Smith \\ Independent Researcher \\ Novara (NO) - 28100 - Italy \\ kira-smith@mil-med.com \\ Received $x x x x x x$ \\ Accepted for publication $x x x x x x$ \\ Published $x x x x x x$
}

\begin{abstract}
SIRTI inhibitors can reduce replication of many viruses with certain similar characteristics to those of Coronaviruses, while $p 53$ protein is another important factor in down-regulation of growth. Tenovin is a class of small molecules that inhibit Sirtuin 1 and 2 , in addition to activate $p 53$ protein, by means of regulation of the interactions used by Coronaviruses as self-defense mechanism. By blocking virus growth and continuous replication, associating the already tested Antiviral medicines, Covid-19 can be definitively eradicated.
\end{abstract}

Keywords: Covid-19, Coronavirus replication, SIRT1 inhibitors, p53, Tenovin

\section{Introduction}

\section{How Coronavirus enters in the host}

To date, it is known that the Novel Coronavirus penetrates into the host via the enzyme converting receptor for angiotensin II (ACE2), by binding to glycoprotein S, with some differences from what happens with Sars-Cov'l

From here, you can draw up a list of interventions to reduce the expression of ACE2 activity, in order to avoid (in whole or in part) the entry of the virus into

the subject under attack, but not only. It is also possible to reduce the ability of the virus to reproduce and lower its endoribonucleic activity. So, let us see what can be done.

\section{ACE2 inhibitors: direct and indirect way}

We assume that ACE inhibitors (Lisinopril, Quinapril, Captopril) don't reduce ACE2 activity, despite being structurally related and traceable in the same organs, ACE2 has opposite biological effects (vasodilatation, bronchodilatation, activity to compensate for physical stresses, as some particular conditions, like hypoxia); some direct ACE2 antagonists are:

- The small molecule MLN-4760-B and its isomeric MILN-4760, much more selective and effective;

- DX600 peptide (and DX-512) having a nanomolar affinity for ACE2, much more than for ACE 
(almost null), competitive and non-competitive inhibition.

The effectiveness and selectivity between these two elements are concentration-related, but at the concentration of $10 \mu \mathrm{M}, \mathrm{MLN}-4760$ was found to have a better activity profile. ${ }^{2}$

SIRTI inhibitors as ACE2 and virus replication suppressor

There is also the possibility to act indirectly, through a Sirtuine group protein, 7 enzymes NAD+ dependent, known as SIRTI.

Expression of ACE2 activity varies with binding to SIRT1 protein, so inhibiting SIRTI, we'll lower ACE2 activity;

Sirtuine are located in the cellular nucleus (SIRT), SIRT6 and SIRT7), in the cytoplasm (SIRT2), or in the mitochondria (SIRT3, SIRT4 and SIRT5) and are involved in many cellular functions, such as metabolism, the cell cycle, l'apoptosis, DNA repair, etc. Recent studies have found that sirtuine may also have enzymatic functions. They are sensors of changes in the intra- and extracellular environment, generally involved in maintaining human health, but are also implicated in some viruses replication.

\section{Method of Investigation}

\section{Examples of virus replication reduced by Sirtuines} inhibitors

- Think, for example, of the Tat protein of the HIV virus: its activity is regulated by SIRT1, whose activity of deacetylase, vice versa is inhibited by the protein Tat. The transcription of HIV is regulated by SIRTI, by means of Tat deacetylase. SIRTI preserves the defenses of the virus, throughout its evolution, allowing the recycling of the protein tat, which binds to
TAR, and the continuous prolongation of the mRNA transcription; ${ }^{4}$

- SIRT1 inhibition (and sometimes even SIRT2) by minor changes to siRNA, is known to cause the decrease in replication of additional viruses: flu strains, VSV (Vesicular Stomatitis Virus), KSHV (Kaposi's Sarcoma-associated Herpesvirus), Hepatitis $\mathrm{B}, \mathrm{HCMV}$, adenovirus, polyomavirus and in some diseases counteract: some types of cancer and Huntington's chorea, sometimes in combination with antivirals, for the best results;

- Among the various other viruses that respond by slowing their growth, there is just Mers-Cov. It has been observed that SIR2 (SIRT1 is the human correspondent) acts as the proviral of Mers-Cov in the yeast, due to interactions between ORF4a and eukaryotic cells. YDL042C/SIR2 yeast gene is a suppressor of ORF4a function. When SIRT1 is inhibited by either chemical or genetic manipulation, there is a reduction of MERS-CoV replication. Moreover, ORF4a inhibited SIRT1-mediated modulation of NF-KB signaling, demonstrating a functional link between ORF4a and SIRTI in mammalian cells. It been identified a functional link between the MERS-CoV ORF4a proteins and the YDL042C/SIR2 yeast gene. ${ }^{5}$

- VSV-SARS-St19 infection is mediated by SARSCoV-S protein in an ACE2-dependent manner. VSVSARS-St19 will be useful for analyzing the function of SARS-CoV-S protein and for developing rapid methods of detecting neutralizing antibodies specific for SARSCoV infection.

SIRTI inhibitors stop the growth of VSV, since that cells' apoptotic response is reduced in cells affected by VSV. ${ }^{\circ}$

\section{Coronavirus self-defense mechanism}

\section{INTERACTION WITH SARS-UNIQUE DOMAIN (SUD) AND PAPAIN-LIKE PROTEASE (PLPRO) AGAINST P53}

The strategy developed from Sars-Cov and other coronaviruses against host immune recognition 
system is expressed by a Sars-Unique Domain (SUD) that interacts with his partner cellular E3 ubiquitin ligase ring-finger and $\mathrm{CHY}$ zinc-finger domain-containing 1 ( $\mathrm{RCHYl)}$ and with papain-like protease (PLPRO). The consequence is a downregulation of $\mathrm{p} 53$, involved in reduction of virus replication. The SARS-CoV papain-like protease is encoded next to SUD within nonstructural protein 3. A SUD-PLPRO fusion interacts with RCHYl more intensively and causes stronger $p 53$ degradation than

SARS-CoV PLPRO alone. ${ }^{7}$

\section{How p53 is very important in Coronavirus} replication

P53 protein reduce coronaviruses replication, because normally activates genes that are involved in the cell's non-specific antiviral defense system. In cells which p53 is too lower, the rate of coronavirus replication is several orders of magnitude higher than that observed in cells in which the p53 is present. ${ }^{7}$

\section{Conclusions}

\section{Tenovin-1 as hypothetic perfect molecule in the halt of replication}

Tenovin-1 [Formal name: N-[[[4(acetylamino)phenyl]amino]thioxomethyl-4-(1,1dimethylethyl)]-benzamide] is a small molecule inhibitor of sirtuin 1 and sirtuin $2,{ }^{8}$ an activator of $p 53$, less toxic of Tenovin-6. Tenovin-1 at concentration of $10 \mu \mathrm{M}$ protects $\mathrm{p} 53$ from $\mathrm{mdm} 2$-mediated degradation with little effect on p53 synthesis. Histone deacetylase inhibitors like tenovin- 1 can also indirectly affect the function of some E3 ubiquitin ligases.

Tenovin-6 is an analog of tenovin-1 At $10 \mu \mathrm{M}$, this compound is slightly more effective than tenovin- 1 at elevating $p 53$ activity, but it's more toxic. 


\section{References}

1 Yun Chen, Yao Guo, Yihang Pan, Zhizhuang Joe Zhao. Structure analysis of the receptor binding of 2019nCoV. Biochemical and Biophysical Research Communications 525, 135-140 Elsevier BV, 2020. Link

2 Wenhui Li, Chengsheng Zhang, Jianhua Sui, Jens H Kuhn, Michael J Moore, Shiwen Luo, Swee-Kee Wong, I-Chueh Huang, Keming Xu, Natalya Vasilieva, Akikazu Murakami, Yaqing He, Wayne A Marasco, Yi Guan, Hyeryun Choe, Michael Farzan. Receptor and viral determinants of SARS-coronavirus adaptation to human ACE2. The EMBO Journal 24, 1634-1643 Wiley, 2005. Link

3 Nicola E. Clarke, Nikolai D. Belyaev, Daniel W. Lambert, Anthony J. Turner. Epigenetic regulation of angiotensinconverting enzyme 2 (ACE2) by SIRT1 under conditions of cell energy stress. Clinical Science 126, 507-516 Portland Press Ltd., 2013. Link

4 Emre Koyuncu, Hanna G. Budayeva, Yana V. Miteva, Dante P. Ricci, Thomas J. Silhavy, Thomas Shenk, Ileana M. Cristea. Sirtuins Are Evolutionarily Conserved Viral Restriction Factors. mBio 5 American Society for Microbiology, 2014. Link

5 Stuart Weston, Krystal L. Matthews, Rachel Lent, Alexandra Vlk, Rob Haupt, Tami Kingsbury, Matthew B. Frieman. A Yeast Suppressor Screen Used To Identify Mammalian SIRTl as a Proviral Factor for Middle East Respiratory Syndrome Coronavirus Replication. Journal of Virology 93 American Society for Microbiology, 2019. Link

6 Shuetsu Fukushi, Rie Watanabe, Fumihiro Taguchi. Pseudotyped Vesicular Stomatitis Virus for Analysis of Virus Entry Mediated by SARS Coronavirus Spike Proteins. 331-338 In Methods in Molecular Biology. Humana Press, 2008. Link

7. Dr. Kira Smith, Tenovin-1 as potential Covid-19 treatment agent, 03/2020

8. Yue Ma-Lauer, Javier Carbajo-Lozoya, Marco Y. Hein, Marcel A. Müller, Wen Deng, Jian Lei, Benjamin Meyer, Yuri Kusov, Brigitte von Brunn, Dev Raj Bairad, Sabine Hünten, Christian Drosten, Heiko Hermeking, Heinrich Leonhardt, Matthias Mann, Rolf Hilgenfeld, Albrecht von Brunn. p53 down-regulates SARS coronavirus replication and is targeted by the SARS-unique domain and PLpro via E3 ubiquitin ligase RCHYl. Proceedings of the National Academy of Sciences 113, E5192-E5201 Proceedings of the National Academy of Sciences, 2016. Link 